\title{
A NEW EXPERIMENTAL PROCEDURE TO OBTAIN TITANIA POWDERS AS ANATASE PHASE BY A SOL-GEL PROCESS
}

Robson Fernandes de Farias*

Departamento de Química, Universidade Federal de Roraima, CP 167, 69301-970 Boa Vista - RR

Recebido em 2/7/01; aceito em 3/4/02

\begin{abstract}
Titania powders were synthesized by a sol-gel process using titanium tetrabutoxide as precursor. The syntheses were performed in water or in solutions of dimethylformamide ( $\mathrm{dmf}$ ) or dimethylsulfoxide (dmso). It is demonstrated, by X-ray diffraction patterns of the synthesized powders, that the samples obtained in $\mathrm{dmf}$ or dmso solutions are crystalline (anatase phase) with some minor amount of brookite phase, whereas the sample synthesized in water is amorphous. The anatase phase can be obtained independently of any previous or further treatment of the synthesized powder, such as hydrothermal or heat treatment, providing a new, simple, quick and inexpensive route to synthesize anatase powders. From the peak broadening of the anatase (101) diffraction, the crystallite sizes were calculated as $6 \mathrm{~nm}$.
\end{abstract}

Keywords: titania; anatase; sol-gel; X-ray diffraction.

\section{INTRODUCTION}

Taking into accont its possible applications as gas sensor, dielectric ceramic or photocalyst ${ }^{1}$, titanium dioxide, $\mathrm{TiO}_{2}$, has been extensively studied. The sol-gel process ${ }^{2}$ has been employed as a successful synthetic approach to obtain titania ${ }^{3}$ or titania-zirconia ${ }^{4}$ powders with spherical morphology.

Titania has three naturally occurring polymorphs named anatase, brookite and rutile ${ }^{5}$. Rutile has been considered the most stable polymorph at standard conditions. Anatase and brookite are considered as kinetic products. On the other hand, depending on the particle size, anatase becomes more stable than rutile ${ }^{6}$.

Many investigations dealing with the structural transitions of solgel synthesized titania powders have been performed, and it has been shown that hydrothermal and heat treatment of amorphous titania ${ }^{7,8}$ can exert remarkable influences on the sttructural transitions.

In such kind of investigations, it has been demonstrated that the sol-gel synthesized titania powders are always amorphous in nature, and that a crystalline structure is achieved only after a heat treatment, at a temperature dependent on the temperature of a previous hydrothermal treatment ${ }^{7,8}$.

The purpose of this publication is to demostrate that titania powders obtained by a sol-gel process performed in dimethylformamide (dmf) or dimethylsulfoxide (dmso) solutions are crystalline (anatase phase), independent of any further or previous hydrothermal or heat treatment, providing a new, simple, quick and inexpensive route to synthesize anatase powders.

Dimethylformamide and dimethylsulfoxide were chosen taking into account their nucleophilic nature, which could exert kinetic effects on the titanium alkoxide hydrolysis.

\section{EXPERIMENTAL}

Hydrolysis and polycondensation of titanium tetrabutoxide (Aldrich) were performed in water and solutions of dimethylformamide (dmf) or dimethylsulfoxide (dmso). In a typical

*e-mail: robdefarias@bol.com.br synthesis, $5 \mathrm{~cm}^{3}$ of titanium tetrabutoxide was added to $50 \mathrm{~cm}^{3}$ of water, dmf or dmso, under stirring. For dmf and dmso systems, 1.5 $\mathrm{cm}^{3}$ of deionized water was added to the solution. The powders were aged for $24 \mathrm{~h}$, and then exaustively washed with deionized water and dried under vacuum at room temperature for $48 \mathrm{~h}$. Infrared spectra indicate that no organic moiety is present in the powders, after the washing operation.

The X-ray diffraction patterns were recorded with a Shimadzu apparatus using $\mathrm{Cu}-\mathrm{Ka}$ radiation.

\section{RESULTS AND DISCUSSION}

The X-ray diffraction patterns of the synthesized samples are shown in Figure 1. As can be observed, titania powders synthesized in $\mathrm{dmf}$ or dmso solutions are crystalline, whereas the sample synthesized in water is amorphous. It is worth noting that the samples are crystalline as synthesized, without needing any previous or further treatment such as heating or hydrothermal aging, as observed for other sol-gel derived titania powders ${ }^{7,8}$. This phenomenon is probably related with the formation of $\mathrm{TiO}_{3}$ groups, which could favour the formation of the anatase phase, with a localized structure with four edges shared by $\mathrm{TiO}_{6}$ octahedral units, rather than the amorphous phase, with only two shared edges ${ }^{9-12}$.

All peaks, including that one around $25^{\circ}$, confirm that anatase is the crystalline phase formed ${ }^{7,8}$. On the other hand, the diffraction peak around $30^{\circ}$ can be attributed to the presence of a minor amount of brookite phase ${ }^{7,8}$. Obviously, the presence of the organic molecules (dmf or dmso) provides new paths for the hydrolysis and polycondensation processes, leading to ordered matrices, instead of amorphous ones. From the peak broadening of the anatase (101) diffraction, the mean crystallite sizes were calculated as $6 \mathrm{~nm}$ (Scherrer's equation).

\section{CONCLUSION}

Since the powders synthesized in dmf or dmso are crystalline in nature independent of any previous or further treatment of the synthesized powder, such as hydrothermal or heat treatment, the experimental procedure proposed is a new, simple, quick and 

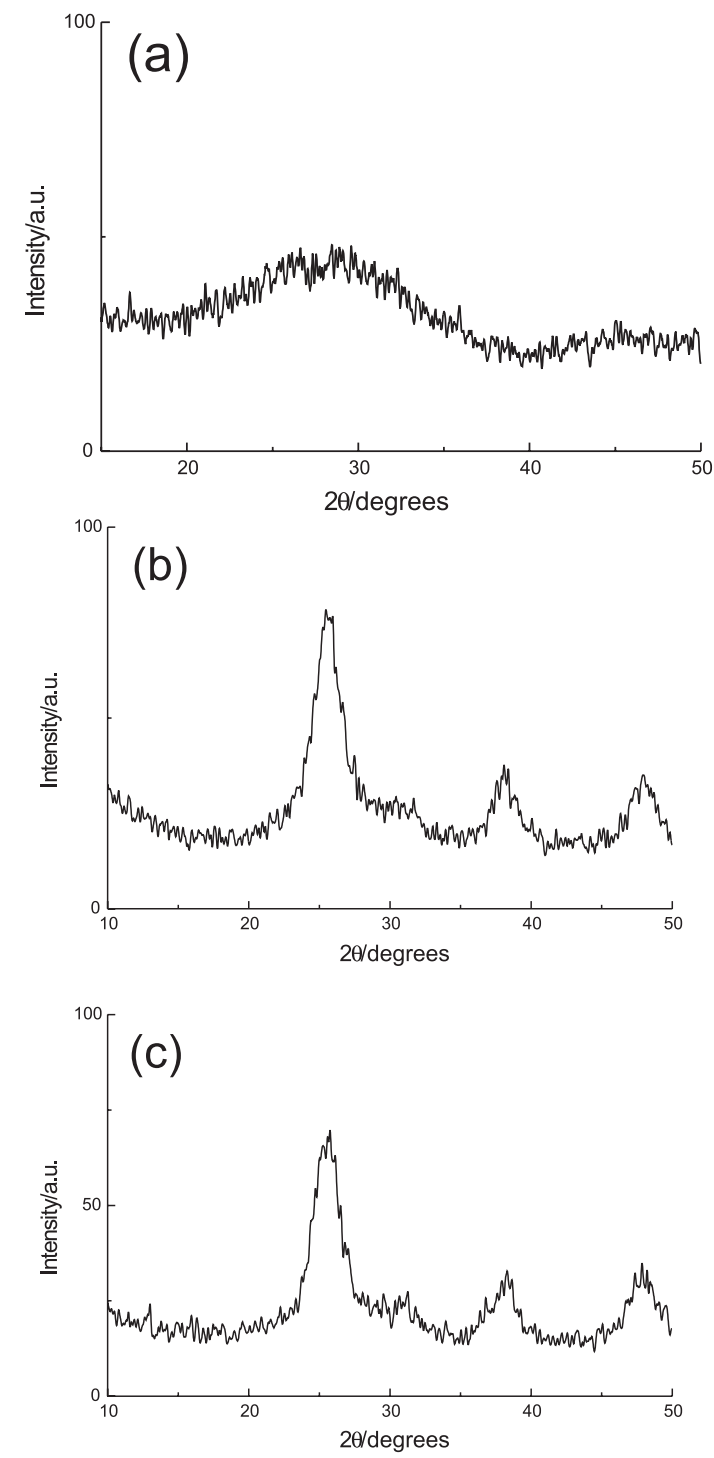

Figure 1. X-ray diffraction patterns for titania powders obtained by a solgel process in water (a), dmf (b) and dmso (c) inexpensive route to synthesize anatase powders. As can be observed, by comparing the X-ray diffraction patterns of the synthesized matrices, anatase is always the crystalline phase obtained, independent on the specific solvent used (dmf or dmso).

\section{ACKNOWLEDGEMENTS}

The author thanks Prof. C. Airoldi, from the Instituto de Química, Unicamp, for the experimental facilities.

\section{REFERENCES}

1. Rao, C.N.R.; Gopalakrishnan, J.; New Directions in Solid State Chemistry, $2^{\text {nd }}$ ed., Cambridge University Press: Cambridge, 1997.

2. Brinker, C.J.; Scherer, G.W.; Sol-Gel Science, Academic Press: New York, 1990.

3. Scolan, E.; Sanchez, C.; Chem. Mater. 1998, 10, 3217.

4. Farias, R.F. de; Airoldi, C.; J. Colloid Interface Sci. 1999, 220, 255.

5. Greenwood, N.N.; Earnshaw, A.; Chemistry of the Elements, ButterworthHeinemann: Oxford, 1995.

6. Zhang, H.; Banfield, J.F.; J. Mater. Chem. 1998, 8, 2073.

7. Ovenstone, J.; Yanagisawa, K.; Chem. Mater. 1999, 11, 2770.

8. Wang, C.-C.; Ying, J.Y.; Chem. Mater. 1999, 11, 3113.

9. Yanagizawa, K.; Yamamoto, Y.; Feng, Q.; Yamasaki, N.; J. Mater. Res. 1998, 13,825 .

10. Bastow, T.J.; Moodie, A.F.; Smith, M.E.; Whitfield, H.J.; J. Mater. Chem 1993, 3, 697.

11. Bastow, T.J; Whitfield, H.J.; Chem. Mater. 1999, 11, 3518

12. Ocana, M.; García-Ramos, J.V.; Serna, C.J.; J. Am. Ceram. Soc. 1992, 75, 2010 . 